\title{
Edema macular en síndrome de Vogt-Koyanagi-Harada tratado con terapia antiangiogénica. Informe de un caso
}

\section{Macular edema in Vogt-Koyanagi-Harada syndrome treated with antiangiogenic therapy. Case report}

\author{
Manuela Alejandra Flores Peraza1*, Pedro Iván González Camarena², Gerardo Martínez del Villar \\ Rita Rios Prado y Ernesto Alejandro Díaz del Castillo Martín ${ }^{3}$
}

${ }^{1}$ Fellowship de la alta Especialidad en Retina y vitreo; ${ }^{2}$ Médico adscrito al servicio de Neurooftalmología; ${ }^{3}$ Médico adscrito al servicio de Retina y Vítreo; ${ }^{4}$ Médico adscrito al servicio de Úvea; ${ }^{3}$ Médico jefe de División de Oftalmología. Hospital de oftalmología, Centro Médico Nacional Siglo XXI, Ciudad de México, México

\section{Resumen}

El 50\% de los pacientes con síndrome de Vogt-Koyanagi-Harada presenta complicaciones oculares; de estos, el 5-10\% presenta membrana neovascular y edema macular, como la paciente que presentamos. Paciente femenino de 39 años de edad con edema macular y síndrome de Vogt-Koyanagi-Harada de un año de evolución. Se aplicaron 2 inyecciones intravitreas de fármacos antiangiogénicos y su evolución fue seguida objetivamente con tomografía de coherencia óptica. Discusión: Las inyecciones intravitreas de antiangiogénicos son una alternativa en el tratamiento del edema macular secundario a enfermedades oculares inflamatorias comparado con la terapia convencional con inyecciones de esteroides subtenonianas e intravítreas.

Palabras clave: Síndrome Vogt-Koyanagi-Harada. Edema macular. Tomografía de coherencia óptica. Terapia antiangiogénica. Ranibizumab.

\section{Abstract}

$50 \%$ of patients with syndrome Vogt-Koyanagi-Harada presents ocular complications, 5-10\% have neovascular membrane and macular edema. We present a 39-year-old woman with macular edema secondary to syndrome Vogt-Koyanagi-Harada, treated with antiangiogenic intravitreal injections and its evolution was objectively followed with optical coherence tomography. Discussion: Antiangiogenic intravitreal injections are an alternative for the treatment of macular edema secondary to inflammatory eye diseases compared to conventional therapy with sub-Tenon steroids and intravitreal injections.

Key words: Vogt-Koyanagi-Harada syndrome. Macular edema. Optical coherence tomography. Antiangiogenic treatment. Ranibizumab.

\section{Correspondencia:}

*Manuela Alejandra Flores Peraza Fecha de recepción: 20-05-2016

E-mail: dra_floresperaza@yahoo.com; $\quad$ Fecha de aceptación: 05-07-2016 alexjanny12@ hotmail.com
Disponible en internet: 04-08-2016 Rev Mex Oftalmol. 2018;xx(xx):1-5 www.rmo.com.mx

0187-4519/@ 2016 Sociedad Mexicana de Oftalmología. Publicado por Permanyer México SA de CV. Este es un artículo Open Access bajo la licencia CC BY-NC-ND (http://creativecommons.org/licenses/by-nc-nd/4.0/). 


\section{Introducción}

El síndrome de Vogt-Koyanagi-Harada, también conocido como síndrome uveomeníngeo, es una panuveítis granulomatosa bilateral y difusa que cursa con desprendimiento de retina seroso y que puede acompañarse de afectación del sistema nervioso central, alteraciones dermatológicas y auditivas ${ }^{1}$.

La evolución de la enfermedad se divide en 4 estadios clínicos: prodrómico, uveítico agudo, de convalecencia y crónico recurrente ${ }^{1}$.

La fase de nuestra paciente, crónica recurrente, tiene lugar a los meses-años después de la fase aguda, interrumpiendo la fase de convalecencia. Esta fase no está presente en todos los pacientes. Se caracteriza por episodios de uveítis anterior granulomatosa frecuentemente resistente a tratamiento esteroideo. De forma infrecuente se asocia a uveítis posterior. Característicamente suelen aparecer nódulos de iris ${ }^{1}$.

Es la fase de las complicaciones crónicas, como cataratas, glaucoma, edema macular, neovascularización coroidea y papilar y membrana neovascular subretiniana ${ }^{2}$.

El $50 \%$ de los pacientes presentan complicaciones que incluyen: formación de cataratas en aproximadamente el $11-38 \%$ de los pacientes; glaucoma en el $40 \%$ de los pacientes, tanto agudo de ángulo cerrado por desplazamiento anterior del complejo iris-cristalino o por la formación de sinequias posteriores y sinequias anteriores periféricas, como glaucoma crónico de ángulo abierto por el uso prolongado de glucocorticoides; membranas neovasculares coroideas en el $5-10 \%$ de los casos, fibrosis subretiniana y atrofia retinocoroidea extensa ${ }^{2}$.

La neovascularización en estos pacientes se produce tanto por la inflamación mantenida, como por la isquemia coroidea ${ }^{2}$.

\section{Presentación del caso}

Paciente femenino de 39 años de edad originaria del estado de Hidalgo, residente de la Ciudad de México, sin antecedentes heredofamiliares de importancia.

Antecedente de síndrome de Vogt-Koyanagi-Harada incompleto tipo ii, presentando desprendimiento de retina seroso bilateral, tinnitus y vértigo; hace un año en tratamiento con azatioprina, ácido fólico y prednisona.

Acude a consulta por presentar nuevamente baja visual de ambos ojos de 2 meses de evolución, acompañado de metamorfopsias, sin síntomas sistémicos.
La agudeza visual (AV) del ojo derecho era 20/200 que mejoraba con estenopeico a 20/100, su presión intraocular era de $12 \mathrm{mmHg}$; en la exploración del segmento anterior encontramos depósitos retroqueráticos finos, no celularidad, anillo de Vossius y cristalino con opacidad subcapsular posterior. El polo posterior mostraba pérdida de brillo foveolar.

En ojo izquierdo, AV de 20/100 que mejoraba con estenopeico a 20/70, segmento anterior con anillo de Vossius, cristalino con opacidad subcapsular posterior. Polo posterior con pérdida de brillo foveolar.

Se realiza tomografía de coherencia óptica (OCT) de ambos ojos antes de instaurar tratamiento (fig. 1).

Dados los hallazgos se aplica inyección intravítrea de $0.5 \mathrm{mg}$ de ranibizumab (Lucentis ${ }^{\circledR}$ ) en ambos ojos, mejorando su AV a las 2 semanas y alcanzando una AV en ojo derecho de 20/70 que mejoraba con estenopeico a 20/50 y en ojo izquierdo 20/70 mejorando a $20 / 40$.

Se realiza OCT de control (fig. 2) y se decide nueva aplicación de dosis de ranibizumab (Lucentis $\left.{ }^{\circledR}\right)$, logrando una mejoría visual de ojo derecho a 20/50 que mejoraba a 20/40 y ojo izquierdo 20/40 mejorando a 20/30.

Después de la aplicación de la inyección de inicio y a las 4 semanas, el grosor retiniano disminuyó de forma significativa (fig. 3).

La evolución de la paciente siempre fue hacia la mejoría, con cambios del edema macular en OCT posterior a la aplicación de antiangiogénicos (fig. 4).

Actualmente, 6 meses después de la instauración de la terapia la paciente presenta la misma $\mathrm{AV}$, sin recaídas y $\sin$ efectos secundarios.

\section{Discusión}

El edema macular se observa clínicamente como un engrosamiento de las capas de la retina a consecuencia del acúmulo anormal de fluidos. Desde el punto de vista fisiopatológico, se debe a la disrupción del equilibrio entre las fuerzas hidrostáticas en los capilares y los gradientes de presión osmótica tisulares, que favorece la difusión de líquidos a los tejidos próximos. El aumento de las proteínas plasmáticas, con el correspondiente incremento de la presión osmótica determina, a su vez, movimientos de fluidos intravasculares, intercelulares e intracelulares. Dentro del ojo, la existencia de la barrera hematorretiniana, cuya parte interna está constituida por los capilares de la retina, favorece, en presencia de alteraciones capilares, el paso 


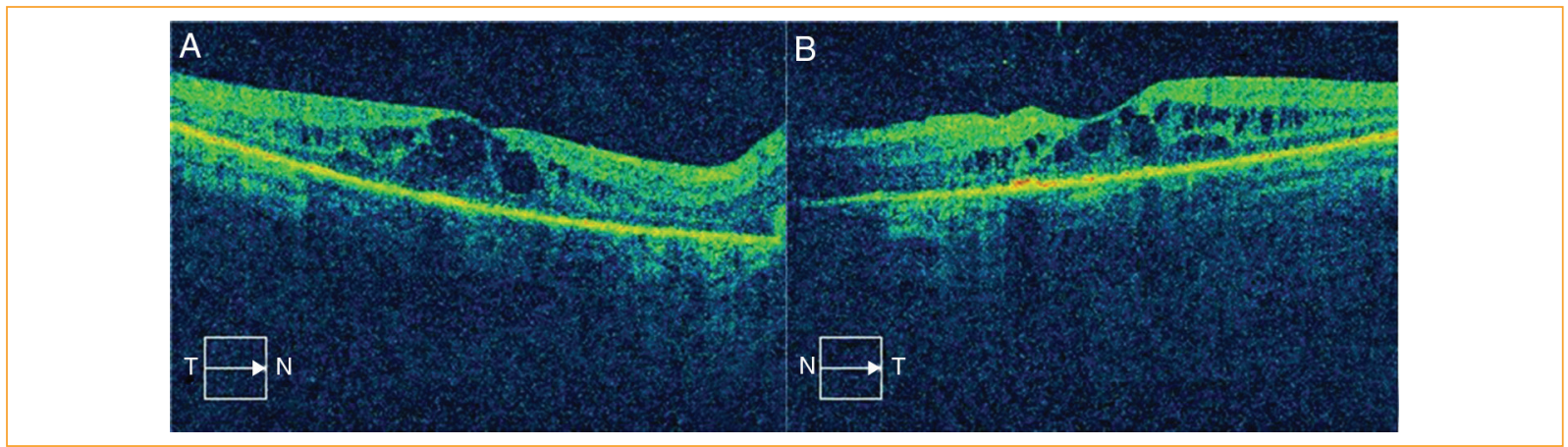

Figura 1. A: OCT OD. Edema macular 426 micras. AV 20/200. Pretratamiento. B: OCT OI. Edema macular 359 micras. AV 20/100. Pretratamiento.

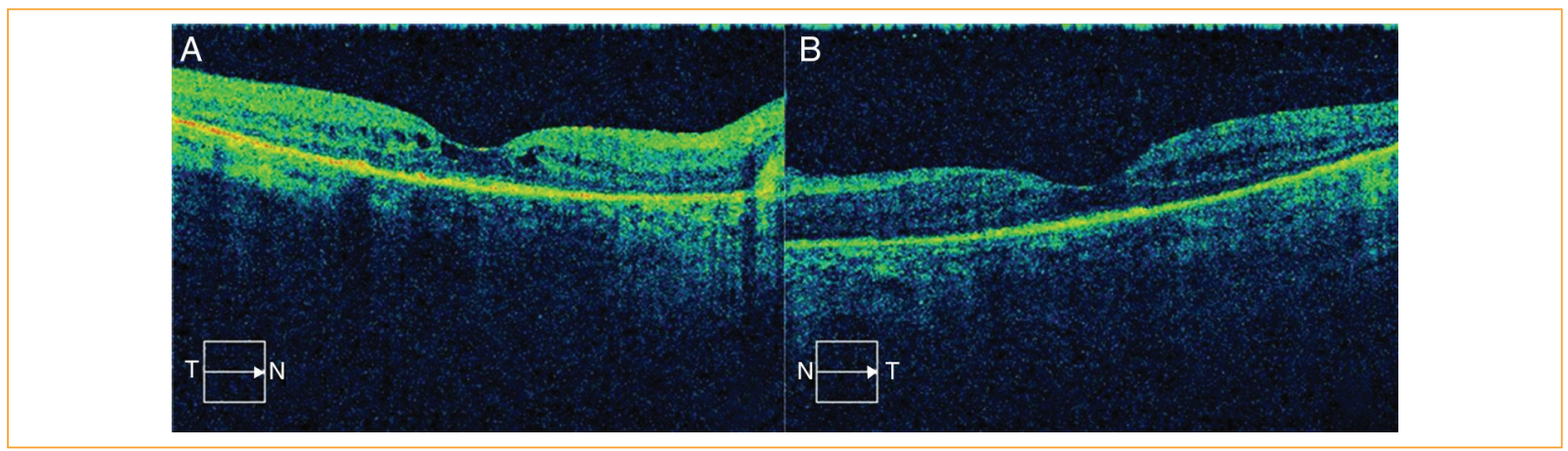

Figura 2. A: OCT OD. Edema macular 238 micras. AV 20/70. Posranibizumab primera dosis. B: OCT OI. Edema macular 209 micras. AV 20/70. Posranibizumab primera dosis.

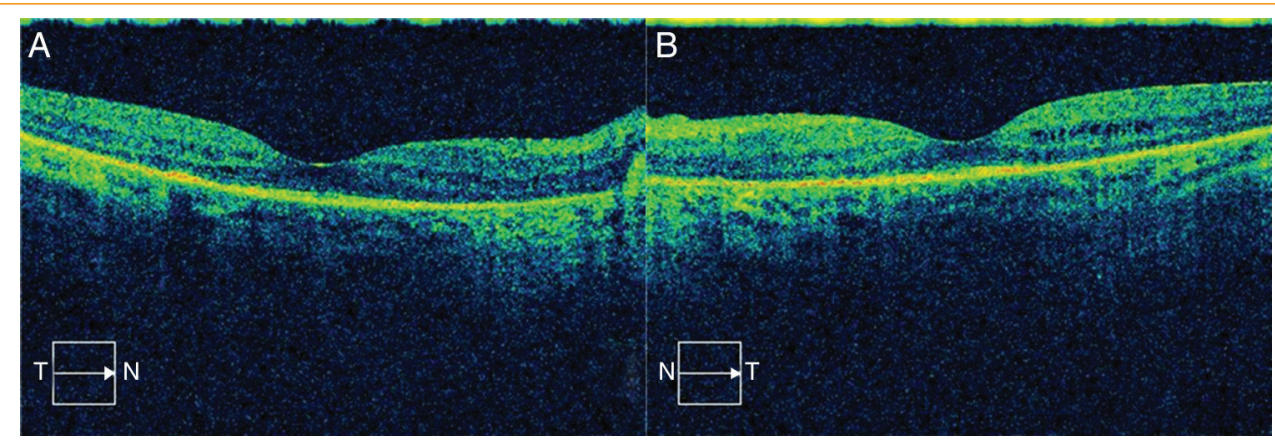

Figura 3. A: OCT OD. Edema macular 211 micras. AV 20/40. Posranibizumab segunda dosis. B: OCT OI. Edema macular 170 micras. AV 20/40. Posranibizumab segunda dosis.

de fluidos en virtud de los cambios hidrostáticos o gradientes osmóticos que existen normalmente ${ }^{3}$.

La permeabilidad vascular anormal de la retina conduce a un edema en la zona de la mácula, que constituye la principal causa de pérdida de la visión en enfermedades como la retinopatía diabética, la degeneración macular exudativa, las oclusiones vasculares retinianas y diversas condiciones inflamatorias y neoplásicas, y aunque una gran variedad de procesos pueden conducir al aumento de la permeabilidad vascular, a través de diferentes mecanismos. El factor de crecimiento endotelial vascular (VEGF) 


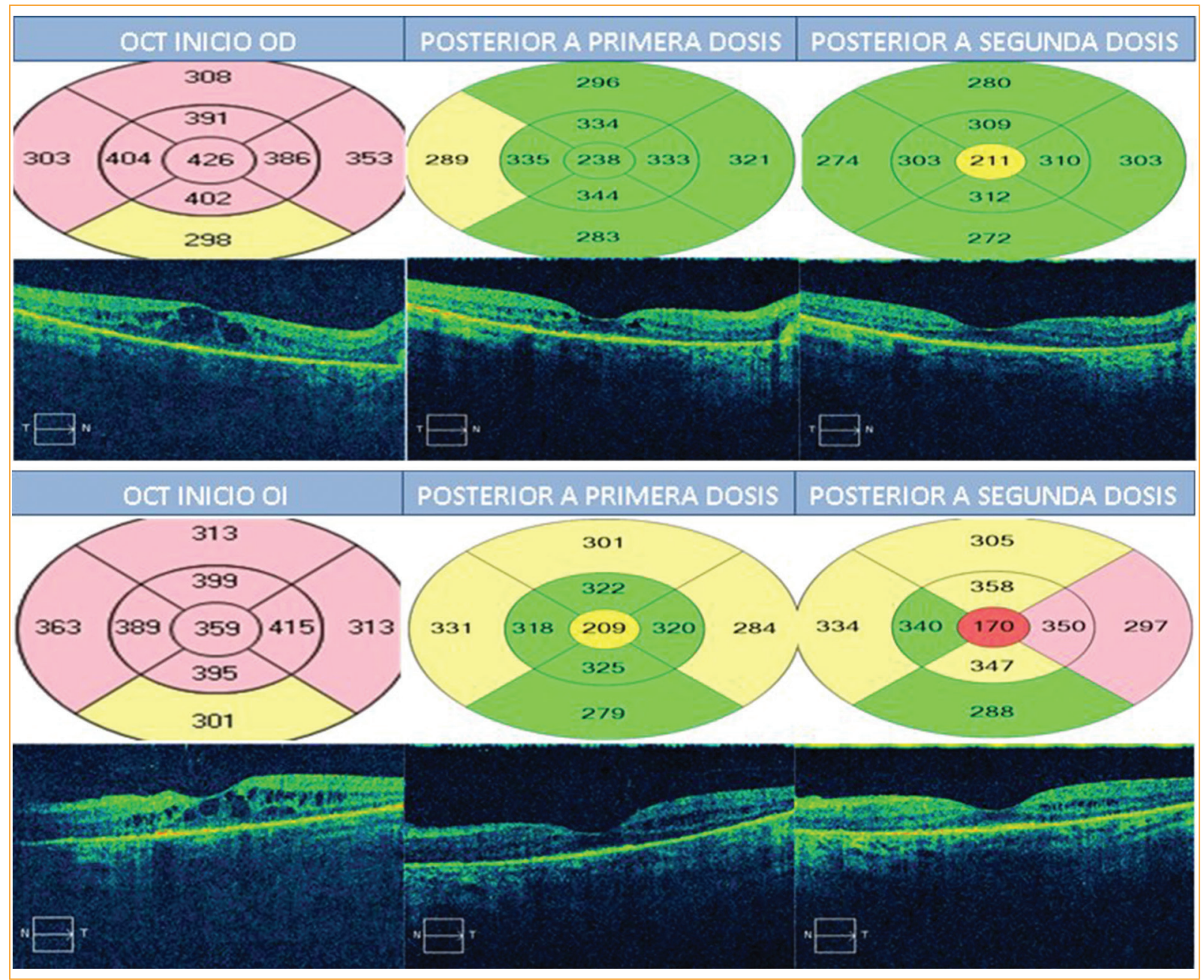

Figura 4. En esta tabla se muestra la evolución del edema macular en ambos ojos, con la medición del grosor macular en la parte media y la imagen tomográfica en los recuadros inferiores. La primera columna muestra las imágenes al inicio del padecimiento cuando se realizó el diagnóstico. La segunda columna muestra las imágenes posteriores a la aplicación de la primera dosis de ranibizumab (Lucentis ${ }^{\circledR}$ ) con menor engrosamiento macular y menor cantidad de espacios quísticos. La tercera columna muestra el resultado final posterior a la segunda aplicación de antiangiogénico.

activa las vías de pérdida vascular, cuyos niveles se correlacionan con una mayor permeabilidad vascular isquémica en las retinopatías y posiblemente también en la degeneración macular exudativa y en la uveítis ${ }^{4}$.

El edema macular es relativamente raro en el síndrome de Vogt-Koyanagi-Harada, en contraste con otros síndromes de inflamación intraocular crónica. Representa una causa rara de pérdida de visión tardía, y puede ser resultado de la fuga de capilares de la retina parafoveal o fuga de líquido a nivel del epitelio pigmentario de la retina.

Una de las piezas clave en la regulación de la angiogénesis es el VEGF y su actividad biológica. Este factor de crecimiento promueve la proliferación de células endoteliales, aumenta la permeabilidad vascular y la inflamación ocular ${ }^{5}$.

EI VEGF es una glucoproteína dimérica que en condiciones de hipoxia es secretada por los pericitos, las células del epitelio pigmentado de la retina y por las células gliales (células de Müller) ${ }^{6}$. EI VEGF estimula directamente el desarrollo de la vasculatura interna y externa del ojo, además actúa como un factor de permeabilidad vascular?. Sus isoformas con actividad biológica son la 121, 165, 189 y 206. Su actividad es mediada por 2 receptores de membrana ligados a tirosina de alta afinidad: el receptor VEGFR1 (FLT1) y el receptor VEGFR2 (FLT2) 8 . 
Por lo general, en condiciones naturales existe un equilibrio entre las moléculas promotoras y las inhibidoras de la angiogénesis. Sin embargo, cuando estas condiciones son alteradas, como sucede durante los episodios de hipoxia o inflamación, este equilibrio se rompe, inclinando la balanza hacia la formación de vasos anormales ${ }^{9}$.

La terapia antiangiogénica moderna se basa en tratar de inhibir la producción 0 , en su defecto, los efectos biológicos de todas las moléculas involucradas, en especial el VEGF ${ }^{10}$.

En nuestra paciente utilizamos como terapia antiangiogénica ranibizumab (Lucentis, Genetech/Novartis ${ }^{\circledR}$ ) que es un fragmento Fab recombinante que une a todas las isoformas activas del VEGF; es sintetizado a partir del bevacizumab. Resulta efectivo para el tratamiento de la neovascularización coroidea secundaria a la degeneración macular relacionada con la edad y para el edema macular. Se encuentra aprobado para su uso clínico desde junio de 2006 ${ }^{11,12}$.

\section{Conclusiones}

Existen estudios que demuestran que tratar este tipo de edema con inyecciones de esteroides subtenonianas e intravítreas mejora la AV en 2 o más líneas de cartilla de Snellen. Los estudios que muestran la eficacia de antiangiogénicos intravítreos obtienen mejoría de al menos 2 líneas; sin embargo, nuestra paciente obtuvo una mejor respuesta con ganancia de 4 líneas y disminución de grosor retiniano de más de 200 micras en ojo derecho y más de 150 micras en ojo izquierdo.

\section{Responsabilidades éticas}

Protección de personas y animales. Los autores declaran que para esta investigación no se han realizado experimentos en seres humanos ni en animales.

Confidencialidad de los datos. Los autores declaran que en este artículo no aparecen datos de pacientes.

Derecho a la privacidad y consentimiento informado. Los autores declaran que en este artículo no aparecen datos de pacientes.

\section{Financiamiento}

Los autores no recibieron patrocinio para llevar a cabo este artículo.

\section{Conflicto de intereses}

Los autores declaran no tener ningún conflicto de intereses.

\section{Bibliografía}

1. Riveros Frutos A, Romera Romero P, Holgado Pérez S, et al. Enfermedad de Vogt-Koyanagi-Harada Semin Fund Esp Reuma-tol. 2012:13:142-6.

2. Tsai JH, Evans M, Rao NA. Comparative study of two sets of criteria for the diagnosis of Vogt-Koyanagi-Harada disease. Am J Ophthalmol. 2006;141:778-9

3. Tranos PG, Wickremasinghe SS, Stangos NT, et al. Macularedema. Surv Ophthalmol. 2004;49:470-90

4. Davis MD, Fisher MR. Diabetic macular oedema. Am J Ophthal-mol. 2003;110:77-82

5. Kowanetz M, Ferrara N. Vascular endothelial growth factor sig-naling pathways: Therapeutic perspective. Clin Cancer Res. 2006;12:5018-22.

6. Madanlou HD, Gharraee Z, Hasan J, et al. Ontogeny of VEGF, IGF-I and $\mathrm{GH}$ in neonatal rat serum, vitreous fluid, and retina from birth to weaning. Invest Ophthalmol Vis Sci. 2006;47:738-44.

7. Kvanta A. Ocular angiogenesis: The role of growth factors. Acta Ophthalmol Scand. 2006:84:282-8.

8. Yoo MH, Hyun HJ, Kob JY, et al. Riluzole inhibits VEGF-inducedendothelial cell proliferation in vitro and hyoeroxia-inducedabnormal vessel formation in vivo. Invest Ophthalmol Vis Sci. 2005:46:4780-7.

9. Hutcheson KA. Retinopathy of prematurity. Curr Opin Ophthal-mol. 2003; $14: 286-90$

10. Manzano RP, Peyman GA, Khan $P$, et al. Testing intravitreal toxi-city of bevacizumab (Avastin). Retina. 2006:26:257-61.

11. Dorrell M, Uusitalo-Jarvinen $\mathrm{H}$, Aguilar E, et al. Ocular neovas-cularization: Basic mechanisms and therapeutic advances. SurvOphthalmol. 2007:52:S3-19.

12. Heier JS, Antoszyk AN, Pavan PR, et al. Ranibizumab for treat-ment of neovascular age-related macular degeneration: A phasei/ii multicenter, controlled, multidose study. Ophthalmology.2006;113:642-4. 